\title{
Effects of the Secular Magnetic Variation on the Distribution Function of Inner-Zone Protons
}

\author{
Thomas A. Farley and Margaret G. Kivelson \\ Department of Planetary and Space Science, Institute of Geophysics and Planetary Physics \\ University of California, Los Angeles, California 9002/4 \\ MarTin WaLt \\ Lockheed Research Laboratory, Palo Alto, California 94304
}

\begin{abstract}
The equation governing the distribution function of high-energy protons in the earth's radiation belt has been modified to include the effects of the secular change of the earth's magnetic dipole moment in addition to the effects of albedo neutron decay, collisional energy loss, and radial diffusion, which have been studied in the past. Because the time scale of the magnetic-field change ( 2000 years) is much longer than the time scale of proton trapping ( $\sim 200$ years) an approximate solution of the equation can be obtained. This solution indicates that the shrinking dipole increases the distribution function at the geomagnetic equator for $1.15<L<1.7$ and $15<E<160 \mathrm{Mev}$. The greatest increase occurs at $L=1.2$, where it is as large as a factor of 5 . The addition of this effect improves the agreement between the theoretically computed distribution function and the experimental data.
\end{abstract}

In a recently published paper, Farley and Walt [1971] argue that the steady-state distribution function of the energetic protons of the inner zone results from a balance between neutron decay additions, collisional losses, and radial diffusion transport. If these are the only mechanisms of importance, some of the higherenergy protons remain trapped for periods of up to several hundred years. Schulz and Paulikas [1972] and Heckman and Lindstrom [1972] have shown independently that the secular change in the earth's magnetic field may have significant effects on protons trapped for such long time periods. In particular, the long-term decrease in the earth's dipole moment produces an electric field that simultaneously convects these protons radially inward and accelerates them to higher energies. This paper adds these additional physical effects to those used earlier to determine the steady-state distribution function. When reasonable assumptions are made, a steady-state distribution function can still be

Publication 1025 of the Institute of Geophysics and Planetary Physics, University of California, Los Angeles, California 90024.

Copyright (c) 1972 by the American Geophysical Union. found. This distribution has been computed and compared with the distribution obtained as a result of neglecting secular variation and with the experimental measurements used earlier by Farley and Walt [1971].

\section{Secular Magnetic-Field Variation}

The average rate of change of the dipole moment of the earth over the last 135 years in which detailed analyses have been made is about $5 \%$ per century, or about $16 \gamma$ per year. Because some of the longer proton residence times are of the order of several hundred years in the inner zone, it is appropriate to use this longterm rate of variation when we compare a theoretical distribution with the presently existing one.

In this paper the rate of change of the magnetic moment of the earth is described as

$$
\left(M_{\circ}\right)^{-1} \frac{\partial M_{0}}{\partial t}=a=-5 \times 10^{-4} \mathrm{year}^{-1}
$$

Because it has been assumed that this rate of change has persisted approximately unchanged for several hundred years, during this time $\operatorname{period} M_{\epsilon}=M_{\mathrm{oe}} e^{a t}$.

Recent satellite data reviewed by Cain [1971] indicate that the rate has increased markedly 
in the last decade to 26 or $27 \gamma$ per year. Whether this increase represents a short-period variation or whether it presages a field reversal is not known.

The nondipole field is changing more rapidly, at a rate of about $50 \gamma$ per year rms. These changes will be shown to have little effect on the present proton distribution in the radiation belt.

\section{Equation for the Distribution Function}

The secular change of the magnetic field is sufficiently slow so that all three adiabatic invariants $M, J$, and $\Phi$ of the trapped protons are conserved in the process. If $f_{1} d M d J d \Phi$ is the number of particles in the volume $d M d J d \Phi$, the distribution function $f_{1}$ remains unchanged when this is the only mechanism acting on the particles,

$$
\left[\partial f_{1}(M, J, \Phi)\right] / \partial t=0
$$

Viewed from fixed spatial coordinates, the protons move inward on drift shells that shrink at a rate determined by the rate of change of the earth's dipole moment and that deform continuously in a manner determined by the changes in the higher multipoles of the field.

The addition of a term representing radial diffusion at constant first and second invariants, two terms representing first and second invariant changes due to collisional energy loss, and a neutron source term yields

$$
\begin{gathered}
\frac{\partial f_{1}(M, J, \Phi ; t)}{\partial t}=\frac{\partial}{\partial \Phi} D_{\Phi \Phi} \frac{\partial f_{1}}{\partial \Phi} \\
-\frac{\partial}{\partial M}\langle\Delta M\rangle f_{1}-\frac{\partial}{\partial J}\langle\Delta J\rangle f_{1} \\
+S_{1}(M, J, \Phi ; t)
\end{gathered}
$$

where $D_{\phi \phi}$ is the diffusion coefficient for thirdinvariant diffusion and the other terms are as defined earlier [Farley and Walt, 1971].

Although the $M, J$, and $\Phi$ coordinates are the most convenient ones in which to establish the equation governing the distribution function, they are not convenient ones in which to solve the equation. The albedo neutron source term and the collisional loss terms depend fundamentally on the momentum of the proton and its location in space. When the secular variation is included, the transformation of momentum and position to $M$ and $\Phi$ coordi- nates is time-dependent, and the right-hand side of (1) will contain terms with explicit time dependence in the $M, J$, and $\Phi$ coordinates. A time-stationary solution will therefore not exist in these coordinates. A transformation of (1) to appropriately chosen coordinates $\mu, J$, and $L$ having a time-independent relation to momentum and geometric space will result in an equation that may have stationary solutions.

The coordinate $L$ is the Mcllwain $L$ value, which, according to its definition, remains at fixed distance from the earth even as the magnetic dipole moment decreases with time. The relation between $L$ and the third invariant $\Phi$ of a trapped proton that is at $L_{0}$ at time $t=0$ is given by

$$
L=\left(2 \pi M_{e}\right) / \Phi=\left(2 \pi M_{0 e} e^{a \iota}\right) / \Phi=L_{0} e^{a t}
$$

where $M_{e}$ is the magnetic dipole moment of the earth having the value $M_{0 e}$ at time $t=0$. The $L$ coordinate of a proton therefore decreases with time according to the relation $d L / d t=a L$.

The new coordinate $\mu$ of each proton is defined in terms of its momentum and a timeindependent magnetic field, and so $\mu=p_{\perp}{ }^{2} /$ $[2 m B(L, t=0)]$. Because the first invariant $M$ is

$$
M=\frac{p_{\perp}{ }^{2}}{2 m B(L, t)}=\frac{p_{\perp}{ }^{2}}{2 m e^{a t} B(L, t=0)}
$$

it follows that the $\mu$ value of a proton will change with time according to the relation $\mu=$ $M e^{a t}$ and $d \mu / d t=a \mu$.

The transformation from momentum and position coordinates to $J$ is not time-dependent, because

$$
\begin{aligned}
& \left.\frac{d J}{d t}\right|_{\mathrm{D}, \mathrm{r} \operatorname{cons} \tan t} \\
& =\frac{d}{d t}\left\{2 p \oint\left[1-\frac{B(s) e^{a t}}{B_{m} e^{a t}}\right]^{1 / 2} d s\right\}=0
\end{aligned}
$$

where the integral is taken between the particle mirror points. As a result, $J$ need not be replaced.

The Jacobian for the coordinate transformation is $\left(2 \pi M_{0 e} / L^{2}\right)$, and so

$$
f_{1}(M, J, \Phi ; t)=\left(L^{2} / 2 \pi M_{00}\right) f_{2}(\mu, J, L ; t)
$$

where $f_{2}(\mu, J, L ; t) d \mu d J d L$ is the number of particles in the volume $d \mu d J d L$. 
Equation 1 expressed in the new coordinates becomes

$$
\begin{aligned}
& \frac{\partial f_{2}(\mu, J, L ; t)}{\partial t}=\frac{\partial}{\partial L} \frac{D_{L L}}{L^{2}} \frac{\partial\left(L^{2} f_{2}\right)}{\partial L} \\
& -\frac{\partial}{\partial \mu}\langle\Delta \mu) f_{2}-\frac{\partial}{\partial J}\langle\Delta J\rangle f_{2}+S_{2}(\mu, J, L) \\
& -\frac{\partial}{\partial L}\left(\frac{d L}{d t} f_{2}\right)-\frac{\partial}{\partial \mu}\left(\frac{d \mu}{d t} f_{2}\right)
\end{aligned}
$$

where

$$
\begin{aligned}
& D_{L L}=\frac{\left\langle(\Delta L)^{2}\right\rangle}{2}=\frac{\left\langle(\Delta \Phi)^{2}(d L / d \Phi)^{2}\right\rangle}{2} \\
&=\left[\frac{L^{2}}{2 \pi M_{0 e} e^{a l}}\right]^{2} D_{\Phi \Phi}
\end{aligned}
$$

The equation governing the distribution function $f_{2}$ has the same form as that governing $f_{1}$ except that two new terms appear, corresponding to the inward convection and energization caused by the dipole shrinkage. In contrast to (1), the equation for $f_{2}$ contains no implicit time dependence in $\langle\Delta \mu\rangle,\langle\Delta J\rangle$, or $S_{2}$ due to dipole shrinkage because $\mu, J$, and $L$ are all obtained by time-independent transformations from momentum and position coordinates. Time-stationary solutions $\left(\partial f_{2} / \partial t=0\right)$ will therefore exist in the $\mu, J$, and $L$ coordinates provided suitable time-independent boundary conditions can be justified.

\section{Effects of Changing Higher Multipoles of the Geomagnetic Field}

A solution in the $\mu, J$, and $L$ coordinates can be transformed to momentum and position coordinates at time $t$ only with the aid of a field representation applicable to time $t$. A particular $L$ shell does not shrink toward the earth, but it does continuously change its state of deformation because of the continuously changing higher multipoles of the earth's field. This pattern will introduce a slight time dependence in $\langle\Delta \mu\rangle$ and $\langle\Delta J\rangle$ because the drift and bounceaveraged atmospheric density on an $L$ shell will change slightly with time. The effect will be most pronounced on the lowest $L$ shells $(L<$ $1.2)$ because here the region of minimum altitude of the proton in its azimuthal drift largely determines the average atmospheric density that it encounters. The residence time of .pro- tons on these low $L$ shells is much shorter $(<10$ years), however, than the characteristic time for change of the geomagnetic field, and these protons cannot have any memory of a different atmospheric density. Where proton residence times are long $(1.3<L<1.7)$ the atmospheric variations due to continuous shell deformation will be very small. This effect has thus been ignored.

Similarly, the source term $S_{2}$ will have some minor time variation even for a time-invariant neutron flux, because the changing deformation of the $L$ shells in space will produce a time variation in the proton injection coefficients [Dragt et al., 1966]. This effect will be small because the neutron source is a weak function of latitude. The effects of the deformation of $L$ shells were ignored by Farley and Walt [1971] in their treatment of the source term for equatorially trapped particles, and in the same spirit those effects are ignored in this work.

\section{BOUNDARY Conditions}

The solution of (2) requires a boundary condition in the $\mu$ and $L$ coordinates at the ends of the range of $L$ in which the equation is assumed to describe the distribution function. In their calculation for the static field Farley and Walt [1971] used the experimental values of the distribution at $L=1.7$, because this is the largest value of $L$ at which time variations have not been observed since the discovery of the radiation belt in 1958. Because their result indicates that some of the highest-energy protons (generally above $100 \mathrm{Mev}$ ) remain in trapped orbits for several hundred years, the validity of their solution at the higher energies requires that the distribution function be constant at $L=1.7$ for that period of time. This constancy cannot be demonstrated and must remain as an assumption of the calculation.

Because of the secular change of the earth's dipole moment it is probable that both the boundary condition $f_{2}(L=1.7)$ and the diffusion coefficient $D_{L_{L}}$ will change on a time scale of 2000 years. Absence of information on these changes is the greatest obstacle to obtaining a time-dependent solution of (2). However, the residence times of most of the protons are so much shorter than 2000 years that it is reasonable to expect the proton distribution to adjust to the boundary conditions and the diffusion 
coefficient more rapidly than the secular variation alters these quantities. Hence the distribution function at any time period can be approximated by a time-independent solution of (2) in which the boundary conditions and the diffusion coefficient appropriate to that time are used.

For these approximate solutions, $t=0$ may be chosen to represent the present epoch, and all quantities in (2) expected to change on a 2000-year time scale may be fixed at their present values, so that

$$
\mu \rightarrow M
$$

$f_{2}(\mu, J, L=1.7 ; t)$

$$
\begin{array}{r}
\rightarrow f_{2}(M, J, L=1.7 ; t=0) \\
D_{L L}(\mu, J, L ; t) \rightarrow D_{L L}(M, J, L ; t=0)
\end{array}
$$

Formally, this procedure is equivalent to expanding the time dependence of these quantities in a power series in at about $t=0$ and discarding all but the zeroth-order term. Except for the energization term, the resulting equation appears identical to (2) with $\mu$ replaced by $M$. The energization term $-(\partial / \partial \mu)[(d \mu)$ $\left.d t) f_{2}\right]$ or, equivalently, $-(\partial / \partial \mu)\left(a \mu f_{2}\right)$ becomes $-(\partial / \partial M)\left(a M f_{2}\right)$ in this approximation.

The resulting equation has been solved for $J=0$ (equatorially trapped protons) with the same experimental boundary condition at $L=$ 1.7 as that used earlier by Farley and Walt [1971]. At the other boundaries the conditions $f_{2}(L=1.10, M)=0$ and $f_{2}(L, M=4000)=$ 0 were employed, also as used earlier.

\section{Results and Discussion}

Equation 2 has been solved by the numerical technique employed earlier [Farley and Walt, 1971] and by use of the same solar-cycle averaged atmospheric density. Representative examples of the resulting distribution function for one of the diffusion coefficients used earlier are illustrated in Figure 1, together with the distribution function computed without including the shrinking dipole effects. The points are experimental data taken from Farley and Walt [1971]. The quantity $j_{\perp}$ is the unidirectional proton flux, in protons $\mathrm{cm}^{-2} \mathrm{sec}^{-1} \mathrm{Mev}^{-1} \mathrm{ster}^{-1}$, perpendicular to the field lines at the geomag-

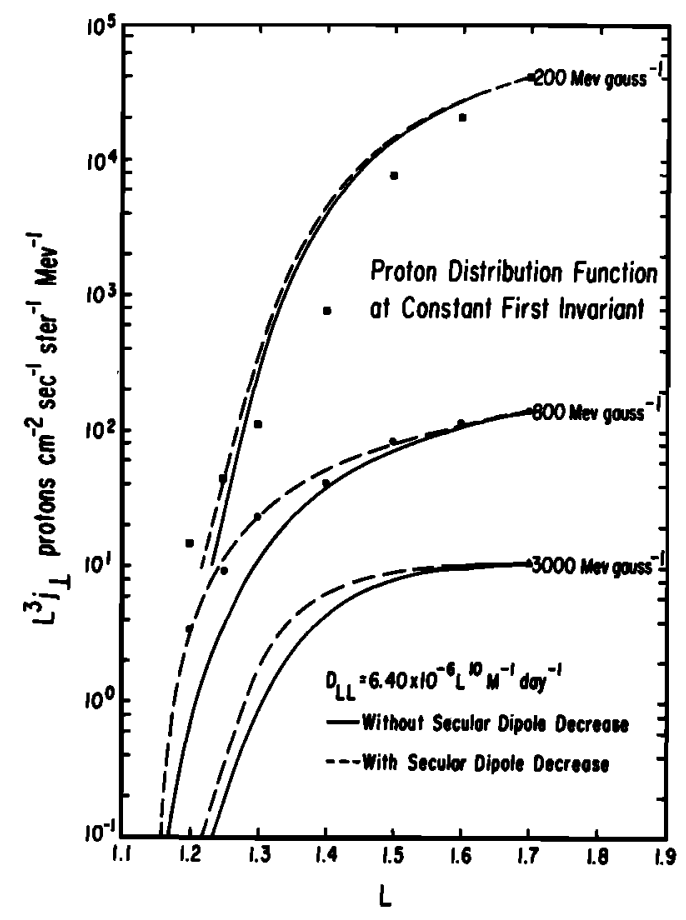

Fig. 1. Representative values of $L^{8} j_{\perp}$ (which is proportional to $L^{2} f$ ) at the geomagnetic equator computed both with and without the decreasing geomagnetic dipole moment. Experimental points [see Farley and Walt, 1971] are included for comparison.

netic equator. Values of $L^{3} j_{\perp}$ have been plotted because $L^{3} j_{\perp}$ is proportional to $L^{2} f_{2}$ for $J=0$.

Curves for 200, 800, and $3000 \mathrm{Mev}$ gauss ${ }^{-1}$ are shown. The extreme values of 200 and 3000 represent the limits of the region used by Farley and Walt [1971] in their study without the secular dipole effects. These extremes are included in Figure 1 to illustrate the effect over as wide a range of $M$ as possible. The curve at $800 \mathrm{Mev}_{\text {gauss }}{ }^{-1}$ is typical of the improvement in the fit that occurs over most of the range of first-invariant $M$. The increase at $L=1.2$ is about a factor of 5 . At the extreme value of $200 \mathrm{Mev}$ gauss $^{-1}$ the effect of the secular dipole change is small, and the fit is not particularly good with or without the new effect. Farley and Walt [1971] attempted with limited success to improve the fit at $200 \mathrm{Mev}$ gauss ${ }^{-1}$ by varying the diffusion coefficient. The present calculation indicates that this discrepancy is not sig- 
nificantly reduced by including the secular dipole variation.

Farley and Walt [1971] had selected the two values $D_{L L}=6.4 \times 10^{-8} L^{10} M^{-1}$ day $^{-1}$ and $1.0 \times$ $10^{-8} L^{10}$ day $^{-1}$ as the best choices for $D_{L_{L}}$ to obtain a fit between the computed distribution function and the experimental data. The increases indicated in Figure 1 suggest the possibility that a smaller radial diffusion coefficient might suffice when the secular variation of the dipole is included. Below $L=1.7$, where the radial gradient of the distribution function is positive, diffusion and dipole decay both contribute to the inward current of protons. However, at any $L$ value the current from dipole decay is proportional to the distribution function, whereas the current from diffusion is proportional to the radial gradient of the distribution function. As a result, it is not possible to offset the effects of a shrinking dipole with a reduction in diffusion coefficient except in a qualitative way.

Table 1 compares the rms deviations of the logarithm of the computed values of $L^{3} j_{1}$ from the logarithm of the data points used earlier by Farley and Walt [1971]; it omits only those at $L=1.7$ (where there are no deviations) and those at $L=1.15$ (where fractional deviations are so large they would completely dominate the result).

The table shows that improvement is significant when the dipole effect is included, regardless of which diffusion coefficient is used. Reducing the coefficients by a factor of 2 produces a slight increase in the rms deviations, thus leaving little reason for preferring either value.

At $L=1.15$, although the new computed values for the distribution function are larger than they were earlier, they are still lower than

TABLE 1. Rms Deviation of the Logarithm of the Distribution Function from the Corresponding Experimental Values Averaged at $L=1.2,1.25,1.3$, 1.4, 1.5, and 1.6 [see Farley and Walt, 1971]

\begin{tabular}{ccc}
\hline & $\begin{array}{c}\text { Without } \\
\text { Dipole Decay, } \\
\%\end{array}$ & $\begin{array}{c}\text { With } \\
D_{L L}, \mathrm{day}^{-1}\end{array}$ \\
\hline $6.4 \times 10^{-8} L^{10} M^{-1}$ & 36 & $\%$ \\
\hline $.2 \times 10^{-6} L^{10} M^{-1}$ & & 16 \\
$1.0 \times 10^{-8} L^{10}$ & 25 & 17 \\
$5.0 \times 10^{-8} L^{10}$ & & 14 \\
\hline
\end{tabular}

the experimental data points by a factor of 10 or more. Farley and Walt [1971] speculated that the deficiencies of the computed distribution function at low $L$ values might be due to solar cycle variations of the atmospheric density whose effects could not be taken into account in a time-stationary theory. The results of this paper indicate that only a minor part of this deficiency can be removed by including the effects of the shrinking dipole. An adequate test of the theory for the lowest $L$ values where proton lifetimes are shorter than the solar cycle will probably require time-dependent solutions, consideration of the possibility that ionospheric current systems affect the radial diffusion, and experimental data with more spatial resolution than has been used here.

A recent measurement [Preszler et al., 1972] of albedo neutrons suggests that in the energy range 10-100 Mev the neutron flux is substantially larger than the estimates used in these calculations. The values of the trapped proton fluxes obtained by (2) and of the inferred radial diffusion coefficients depend on the magnitude of the neutron source term $S_{2}$, and a larger source might improve agreement between theory and experiment in some regions of space. However, the principal result of the present work, namely that the weak variation of the geomagnetic field causes an increase in the trapped proton flux at low $L$ values, is qualitatively independent of the albedo neutron flux.

\section{Conclusions}

The secular decrease of the dipole moment of the earth contributes significantly to the high-energy proton population of the inner radiation belt, increasing the distribution function everywhere. The increases, which are most pronounced at low $L$ values and high magnetic moments, bring about better agreement between experiment and a theory that attributes the protons to the mechanisms of cosmic-ray albedo neutron decay, radial diffusion, and collisional energy loss to the ambient atmosphere.

Acknowledgment. This work was supported at UCLA by NASA grant NGL 05-007-004.

The Editor thanks H. H. Heckman and M. Schulz for their assistance in evaluating this paper. 


\section{REFERENCES}

Cain, J. C., Geomagnetic models from satellite surveys, Rev. Geophys. Space Phys., 9, 259, 1971.

Dragt, A. J., M. M. Austin, and R. S. White, Cosmic ray and solar proton albedo neutron decay injection, J. Geophys. Res., 71, 1293, 1966.

Farley, T. A., and M. Walt, Source and loss processes of protons of the inner radiation belt, J. Geophys. Res., 76, 8223, 1971.

Heckman, H. H., and P. J. Lindstrom, Response of trapped particles to a collapsing dipole moment, J. Geophys. Res., 77, 740, 1972.

Preszler, A. M., G. M. Simnett, and R. S. White, Earth albedo neutrons from 10 to $100 \mathrm{Mev}$, Phys. Rev. Lett., 28, 982, 1972.

Schulz, M., and G. A. Paulikas, Secular magnetic variation and the inner proton belt, J. Geophys. Res., 77, 744, 1972.

(Received April 12, 1972;

accepted July 24, 1972.) 